\begin{tabular}{|c|l|}
\hline Title & $\begin{array}{l}\text { Mutual Inhibition between Carvedilol Enantiomers during Racemate Glucuronidation Mediated by Human Liver and } \\
\text { Intestinal Microsomes }\end{array}$ \\
\hline Author(s) & Takekuma, Y oh; Y agisawa, Keiji; Sugawara, Mitsuru \\
\hline Citation & $\begin{array}{l}\text { Biological and Pharmaceutical Bulletin, 35(2), 151-163 } \\
\text { https://doi.org/10.1248/ppb.35.151 }\end{array}$ \\
\hline Issue Date & 2012-02 \\
\hline Doc URL & http:/hdl.handle.net/2115/48520 \\
\hline Type & article \\
\hline File Information & BPB35-2_151-163.pdf \\
\hline
\end{tabular}

Instructions for use 


\title{
Mutual Inhibition between Carvedilol Enantiomers during Racemate Glucuronidation Mediated by Human Liver and Intestinal Microsomes
}

\author{
Yoh Takekuma,* Keiji Yagisawa, and Mitsuru Sugawara \\ Laboratory of Pharmacokinetics, Department of Biopharmaceutical Sciences and Pharmacy, Faculty of Pharma- \\ ceutical Sciences, Hokkaido University; Kita-12, Nishi-6, Kita-ku, Sapporo 060-0812, Japan. \\ Received August 1, 2011; accepted November 22, 2011; published online November 25, 2011
}

\begin{abstract}
Carvedilol is administered orally as a racemic mixture of $R(+)$ - and $S(-)$-enantiomers for treatment of angina pectoris, hypertension and chronic heart failure. We have reported that enzyme kinetic parameters for carvedilol glucuronidation by human liver microsomes (HLM) differed greatly depending on the substrate form, namely, racemic carvedilol and each enantiomer. These phenomena were thought to be caused by mutual inhibition between carvedilol enantiomers during racemate glucuronidation. The aim of this study was to clarify the mechanism of these phenomena in HLM and human intestinal microsomes (HIM) and its relevance to uridine 5'-diphosphate (UDP)-glucuronosyl transferase (UGT) 1A1, UGT2B4 and UGT2B7, which mainly metabolize carvedilol directly in phase II enzymes. HLM apparently preferred metabolizing $(S)$-carvedilol to $(R)$-carvedilol in the racemate, but true activities of HLM for both glucuronidation were approximately equal. By determination of the inhibitory effects of $(S)$-carvedilol on $(R)$-carvedilol glucuronidation and vice versa, it was shown that $(R)$-carvedilol glucuronidation was more easily inhibited than was $(S)$-carvedilol glucuronidation. UGT2B7 was responsible for $(S)$-carvedilol glucuronidation in HLM. Ratios of contribution to $(R)$-carvedilol glucuronidation were approximately equal among UGT1A1, UGT2B4 and UGT2B7. However, enzyme kinetic parameters were different between the two lots of HLM used in this study, depending on the contribution ratio of UGT2B4, in which $(R)$-glucuronidation was much more easily inhibited by $(S)$-carvedilol than was $(S)$-glucuronidation by $(R)$-carvedilol. Meanwhile, HIM preferred metabolizing $(R)$-carvedilol, and this tendency was not different between the kinds of substrate form.
\end{abstract} tion

Key words carvedilol; uridine $5^{\prime}$-diphosphate-glucuronosyl transferase; glucuronidation; enantiomer; interac-

Many drugs are administered orally as racemic mixtures because of the difficulty or high cost of selective synthesis of one optical isomer. A pair of enantiomers has the same physicochemical properties including melting point, boiling point and solubility without optical rotation. Both enantiomers have been thought to be recognized by the same enzyme in a metabolic process because of the similarity of their chemical structures. However, these enantiomers are recognized as different chemical compounds in vivo, and pharmacological and pharmacokinetic properties often differ between enantiomers. ${ }^{1)}$ In fact, there have been several reports of interaction between enantiomers via a metabolic enzyme., 2 ,3)

Carvedilol is an antihypertensive and antianginal drug that favorably combines $\beta$-adrenergic blocking and vasodilating activities as a result of $\alpha$-blocking action. ${ }^{4,5)}$ Carvedilol is also administered orally as a racemic mixture of $R(+)$ - and $S(-)$ enantiomers.

It is known that carvedilol is metabolized through multiple pathways. The enzymes that are involved in its metabolism are cytochrome P450 (CYP) 2D6 and CYP2C9 as phase I enzymes and uridine 5'-diphosphate (UDP)-glucuronosyl transferase (UGT) 1A1, UGT2B4 and UGT2B7 as phase II enzymes. ${ }^{6,7)}$

CYP2D6 and CYP2C9 have been reported to metabolize $(S)$-carvedilol more rapidly than $(R)$-carvedilol. ${ }^{6)}$ Several studies have shown that the activity of CYP2D6 has a greater effect on the pharmacokinetics of carvedilol than does the activity of CYP2C9 and that a poor metabolizer form of CYP2D6 eliminated carvedilol from serum more slowly than did an extensive metabolizer form though this difference was not observed in CYP2C9. ${ }^{8-11)}$

On the other hand, stereoselective metabolism of carvedilol by the three isoforms of UGT has been reported by several groups. ${ }^{7,12,13)}$ UGT1A1 prefers metabolizing $(R)$-carvedilol to $(S)$-carvedilol, while UGT2B7 prefers $(S)$-carvedilol. In the case of UGT2B4, the rate of $(S)$-carvedilol glucuronidation is slightly higher than that of $(R)$-carvedilol. Moreover, we have reported that mutation of G71R in UGT1A1 and mutations of A71S and H268Y in UGT2B7 decrease the activity of carvedilol glucuronidation in an in vitro assay and that polymorphism of $U G T 2 B 7 * 3(211 \mathrm{G}>\mathrm{T}, \mathrm{A} 71 \mathrm{~S})$ decreases clearance of carvedilol in patients with chronic heart failure. ${ }^{12,14)}$

We have also reported that the rate of $(S)$-carvedilol glucuronidation by pooled human liver microsomes (HLM) was much higher than that of $(R)$-carvedilol when racemic carvedilol was used as a substrate but that a difference between the rates of $(R)$ - and $(S)$-carvedilol glucuronidation was not observed when each enantiomer was used as a substrate. ${ }^{12)}$ Moreover, we have demonstrated that values of $V_{\max }$ and $V_{\max } / K_{\mathrm{m}}$ of both $(R)$ - and $(S)$-carvedilol glucuronidation by HLM in the case of each enantiomer as a substrate are much higher than those in the case of racemic carvedilol and that $(R)$ - and $(S)$-carvedilol might mutually inhibit the glucuronidation. $^{12)}$

The aim of this study was to clarify whether these phenomena have generality and are observed in HLM and pooled human intestinal microsomes (HIM) and to elucidate the details of mutual inhibition between carvedilol enantiomers during racemate glucuronidation mediated by HLM and HIM and its relevance to UGT1A1, UGT2B4 and UGT2B7.

\section{MATERIALS AND METHODS}

Chemicals and Reagents $( \pm)$-Carvedilol was kindly 


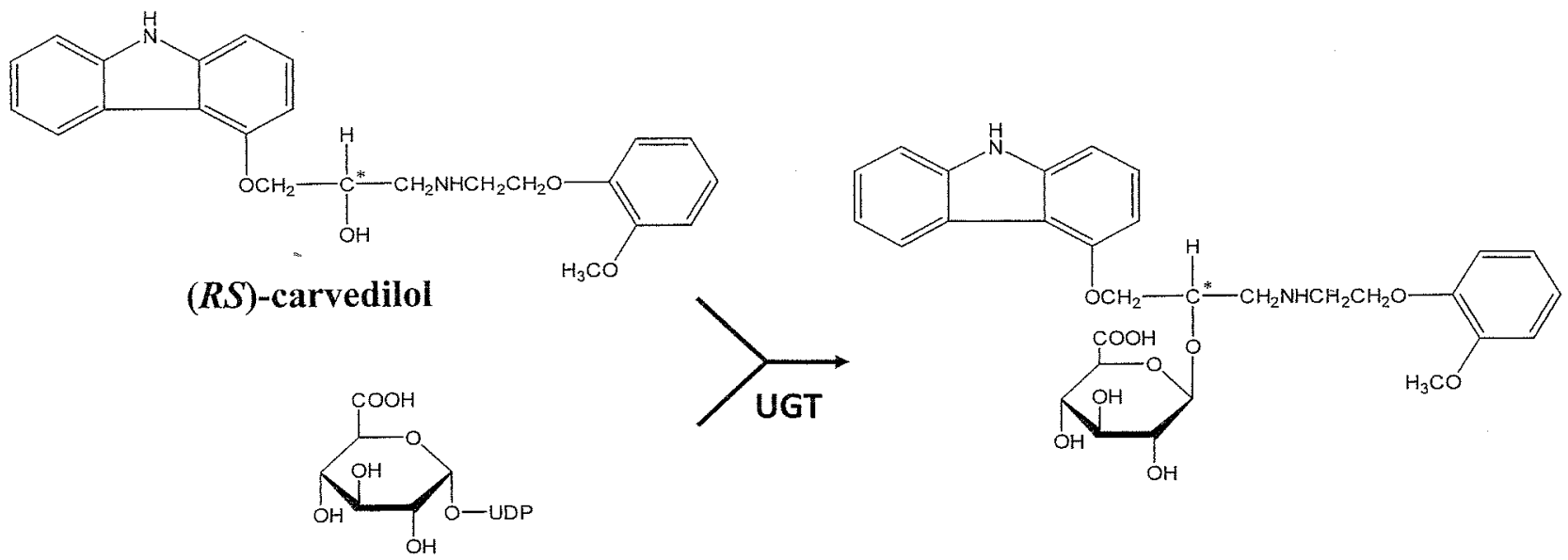

\section{$\alpha$-UDP-glucuronic acid}

Fig. 1. Glucuronidation Reaction of Racemic Carvedilol

supplied by DAIICHI SANKYO Co. (Tokyo, Japan). $R(+)$ carvedilol and $S(-)$-carvedilol were purchased from Toronto Research Chemicals Inc. (Ontario, Canada). Alamethicin and mefenamic acid were obtained from Sigma-Aldrich (St. Louis, MO, U.S.A.). UDP-glucuronic acid trisodium salt was purchased from Wako Pure Chemicals (Osaka, Japan). Pooled human liver microsomes (HLM) (Product. No. X008011 as Lot B and X008012 as Lot A in this study) and pooled human intestinal microsomes (HIM) (Product. No. X02801) were purchased from In Vitro Technologies, Inc. (Baltimore, Maryland, U.S.A.). Microsomes of $\mathrm{S} 9$ cells expressing recombinant UGT1A1, UGT2B4 and UGT2B7 (SUPERSOMES ${ }^{\circledR}$ ) were purchased from BD GENTEST (Franklin Lakes, NJ, U.S.A.). All other reagents were of the highest grade available.

Assay for Carvedilol Glucuronidation Carvedilol glucuronidation using HLM was assayed according to our previous study. ${ }^{12)}$ The reaction mixture contained carvedilol, $0.05 \mathrm{mg} /$ $\mathrm{mL}$ microsomal proteins (HLM, HIM, recombinant UGT1A1, UGT2B4, and UGT2B7), $10 \mathrm{~mm} \mathrm{MgCl} 2,2 \mathrm{mM}$ UDP-glucuronic acid, and $12.5 \mu \mathrm{g} / \mathrm{mL}$ alamethicin in $50 \mathrm{~mm}$ Tris $-\mathrm{HCl}$ buffer $(\mathrm{pH} 7.4)$ in a final volume of $80 \mu \mathrm{L}$. After preincubation at $37^{\circ} \mathrm{C}$ for $5 \mathrm{~min}$, the reaction was initiated by the addition of UDP-glucuronic acid. The mixture was incubated for $0.5-3 \mathrm{~h}$ at $37^{\circ} \mathrm{C}$. Then the reaction was terminated by the addition of $80 \mu \mathrm{L}$ of acetonitrile and $40 \mu \mathrm{L}$ of methanol containing $20 \mu \mathrm{g} /$ $\mathrm{mL}$ flecainide acetate as an internal standard. After removal of the protein by centrifugation at $14000 \times \mathrm{g}$ for $10 \mathrm{~min}$ at $4^{\circ} \mathrm{C}$, $120 \mu \mathrm{L}$ of chloroform was added to $120 \mu \mathrm{L}$ of the supernatant to remove unreacted carvedilol. The mixture was vortexed for $2 \mathrm{~min}$ and was then centrifuged at $14000 \times \mathrm{g}$ for $5 \mathrm{~min}$ at $4^{\circ} \mathrm{C}$. Forty micro liters of the supernatant were injected into an HPLC system.

Inhibitory assays were performed using $(R)$-carvedilol $(0.2-50 \mu \mathrm{M})$ in the case of $(S)$-carvedilol as a substrate, $(S)$ carvedilol $(0.2-50 \mu \mathrm{M})$ in the case of $(R)$-carvedilol as a substrate, or mefenamic acid $(1-500 \mu \mathrm{M})$, and other conditions were the same.

Drug Assay A drug assay was carried out by reversedphase high-performance liquid chromatography (HPLC) with a fluorometric detector according to our previous study with minor modification. ${ }^{12)}$ Namely, the separation was per- formed on a L-column ODS $(4.6 \mathrm{~mm}$ i.d. $\times 150 \mathrm{~mm}$, Chemicals Evaluation and Research Institute, Tokyo, Japan). For quantification of two carvedilol glucuronides derived from $(R)$ - and $(S)$-carvedilol, the mobile phase was a mixture of methanol and $50 \mathrm{~mm}$ potassium dihydrogenphosphate $(44: 56)$ and column temperature was $50^{\circ} \mathrm{C}$. The flow rate was $1.0 \mathrm{~mL} / \mathrm{min}$. Excitation and emission wavelengths of $240 \mathrm{~nm}$ and $340 \mathrm{~nm}$, respectively, were used for fluorometric detection.

Carvedilol was used as the standard assuming the same fluorescence intensities of carvedilol and its glucuronide because glucuronic acid connected site of carvedilol was not related with resonance (Fig. 1).

Western Blot Analysis HLM and HIM were separated by $10 \%$ sodium dodecyl sulfate (SDS)-polyacrylamide gel electrophoresis. The gel was transferred onto a nitrocellulose membrane and probed with mouse anti-human UGT (1A1 or 2B7) antisera (1:500 dilution). An anti-mouse immunoglobulin $G$ (IgG) goat antibody conjugated with horseradish peroxidase was used as the second antibody, and the resulting immunocomplexes were visualized using enhanced chemiluminescence (ECL) Western blotting detection reagents (GE Health Care Bio-Sciences KK, Tokyo, Japan) and exposed on $\mathrm{X}$-ray films.

Data Analysis Data are expressed as means \pm S.D. in figures and tables. Normally distributed measurements were compared by the Student's $t$-test and nonnormally distributed measurements by the Cochran-Cox test. The kinetic parameters were calculated using OriginPro. ver. 8.1 (OriginLab Corp.) by curve fitting to the following equations:

Michaelis-Menten equation

$$
V=\frac{V_{\max } \times[\mathrm{S}]}{K_{\mathrm{m}}+[\mathrm{S}]}
$$

where $V$ is velocity of glucuronidation, [S] is concentration of the substrate, $V_{\max }$ is maximum velocity of glucuronidation, and $K_{\mathrm{m}}$ is Michaelis constant.

Two-enzyme Michaelis-Menten equation

$$
V=\frac{V_{\max 1} \times[\mathrm{S}]}{K_{\mathrm{m} 1}+[\mathrm{S}]}+\frac{V_{\max 2} \times[\mathrm{S}]}{K_{\mathrm{m} 2}+[\mathrm{S}]}
$$

Michaelis-Menten and nonsaturated component equation 
Concentration dependency

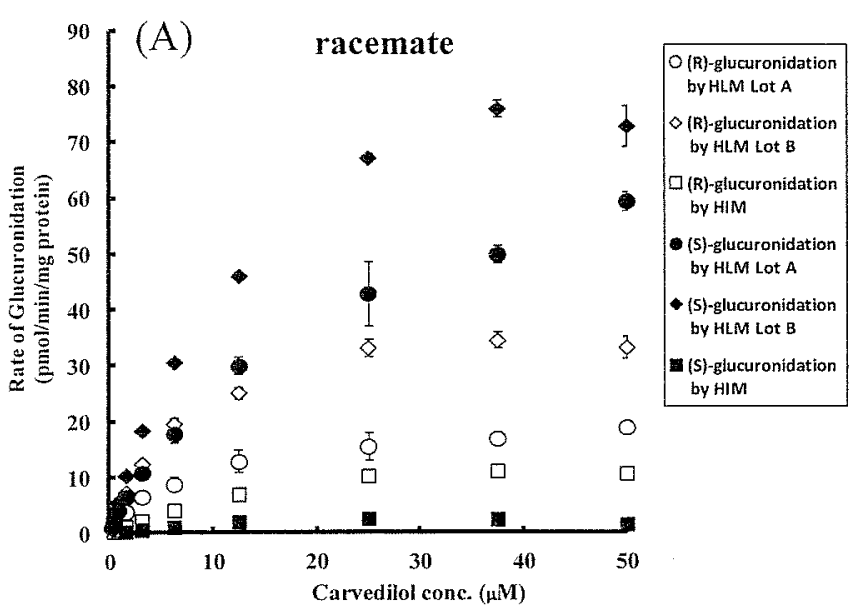

(B)

enantiomer

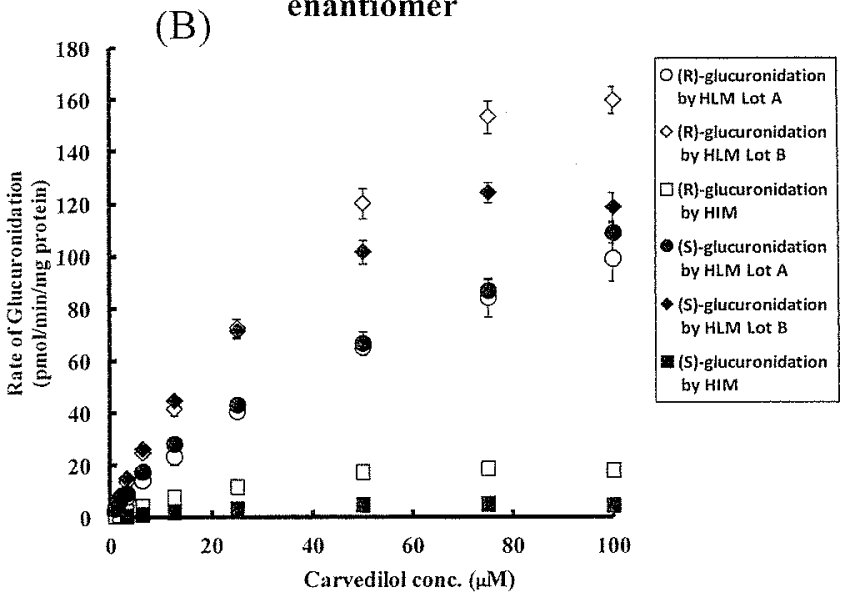

Eadie-Hofstee plot
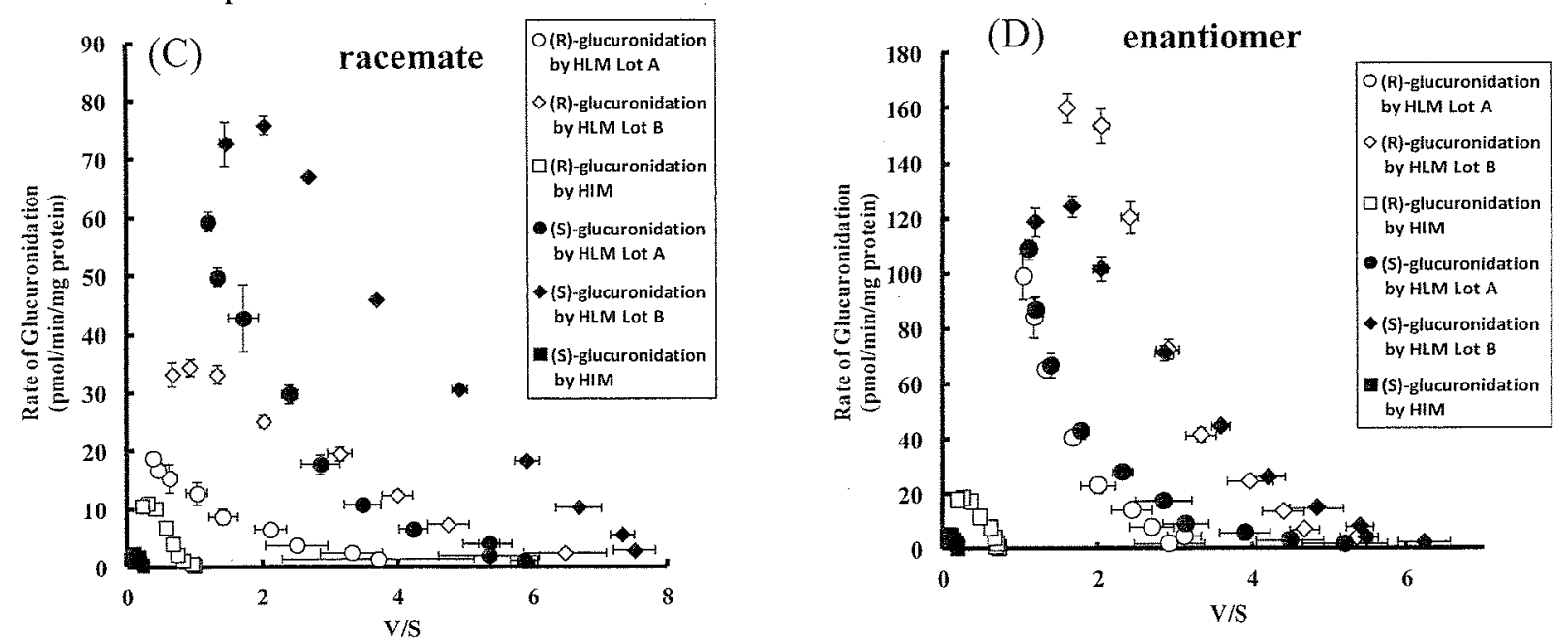

Fig. 2. Concentration Dependency (Top) and Eadie--Hofstee Plot (Bottom) of Glucuronidation Rate of (R)- and (S)-Carvedilol by HLM Lot A, Lot $B$ and HIM

Racemate was used as a substrate in the left graph, and each enantiomer was used as a substrate in the right graph. Racemic carvedilol (0.2- $50 \mu \mathrm{M}$ as a single enantiomer concentration) and $(R)$ - or $(S)$-carvedilol $(0.4-100 \mu \mathrm{m})$ was incubated for $30 \mathrm{~min}$. Each point represents the mean $\pm S . D$. of three independent measurements.

$$
V=\frac{V_{\max } \times[\mathrm{S}]}{K_{\mathrm{m}}+[\mathrm{S}]}+\mathrm{a} \times[\mathrm{S}]
$$

where ' $a$ ' is a constant that is involved in the nonsaturated component.

Construction of Competitive and Noncompetitive Mutual Inhibition Michaelis-Menten Equations Since a racemate was used as a substrate, a mutual inhibition model and enzyme velocity equation were constructed according to Segel's method. Namely, the generally used competitive inhibition model (Eq. 4) and non-competitive inhibition model (Eq. 5) were slightly modified. If one enantiomer inhibits enzyme reaction of the other enantiomer in the racemate, inhibitor concentration [I] is equal to substrate concentration [S] in Eqs. 4 and 5 . Inhibition constant $K_{\mathrm{i}}$ is approximated to $K_{\mathrm{m}}$ of the antipode $\left(K_{\mathrm{m} \text { antipode }}\right.$ ). In this study, Eqs. 6 and 7 , competitive and non-competitive mutual inhibition models, respectively, were derived from this hypothesis.

Competitive inhibition Michaelis-Menten equation:

$$
V=\frac{V_{\max } \times[\mathrm{S}]}{\left(1+\frac{[\mathrm{I}]}{K_{\mathrm{i}}}\right) \times K_{\mathrm{m}}+[\mathrm{S}]}
$$

where $[I]$ is concentration of the inhibitor and $K_{\mathrm{i}}$ is inhibition constant.

Non-competitive inhibition Michaelis-Menten equation:

$$
V=\frac{V_{\max } \times[\mathrm{S}]}{\left(1+\frac{[\mathrm{I}]}{K_{\mathrm{i}}}\right) \times\left(K_{\mathrm{m}}+[\mathrm{S}]\right)}
$$

Competitive mutual inhibition Michaelis-Menten equation:

$$
V=\frac{V_{\max } \times[\mathrm{S}]}{\left(1+\frac{[\mathrm{S}]}{K_{\text {mantipode }}}\right) \times K_{\mathrm{m}}+[\mathrm{S}]}
$$

Non-competitive mutual inhibition Michaelis-Menten equation: 
(a) HLM Lot A

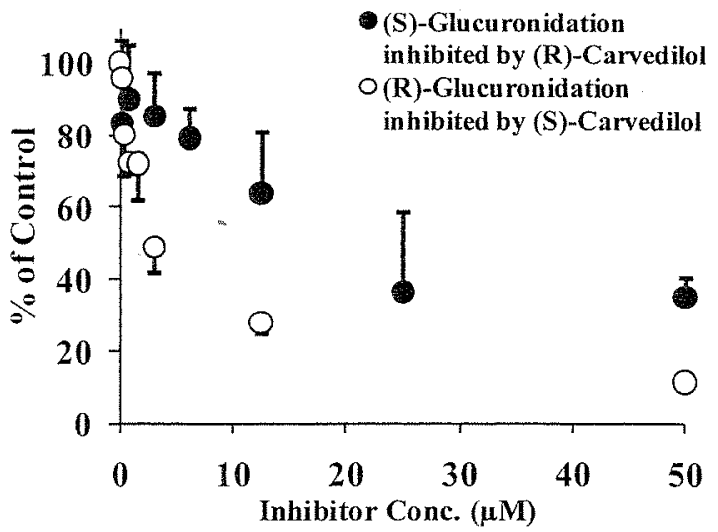

(b) HLM Lot B

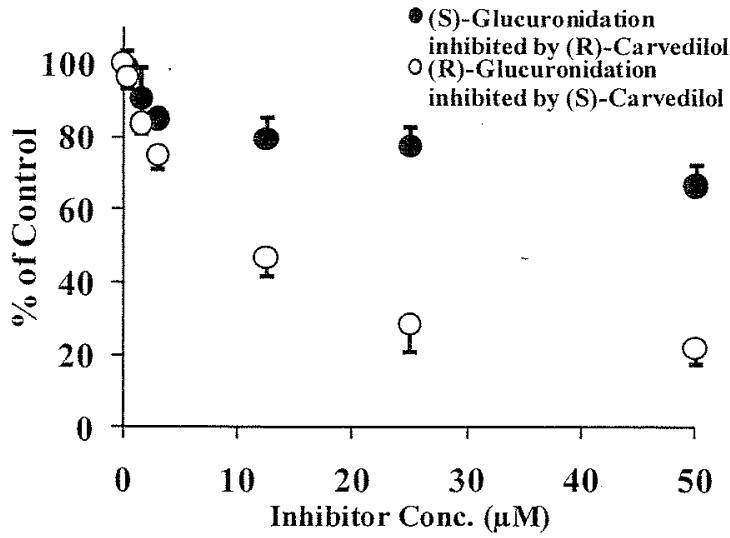

inhibited by (R)-Carvedilol
(S)-Glucuronidation

$O(R)$-Glucuronidation

inhibited by (S)-Carvedilol

(c) HIM

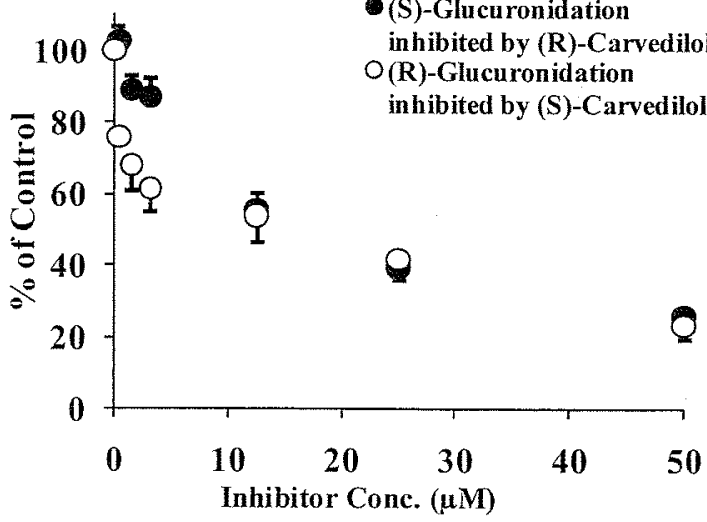

Fig. 3. Inhibitory Effects of Carvedilol Enantiomer on Glucuronidation of Its Antipode by HLM Lot A (a), Lot B (b), and HIM (c)

$(R)$ - or $(S)$-carvedilol $(0.5 \mu \mathrm{M})$ as a sustrate was incubated in the absence and presence of its antipode $(0.2-50 \mu \mathrm{M})$ for $30 \mathrm{~min}$ (HLMs) and $1 \mathrm{~h}$ (HIM). Each point represents the mean \pm S.D. of three to four independent measurements.

Table 1. Kinetic Parameters of Carvedilol Glucuronidation by HLM Lot A, Lot B, and HIM

\begin{tabular}{|c|c|c|c|c|c|c|c|}
\hline \multirow[b]{2}{*}{$\begin{array}{l}\text { Enzyme } \\
\text { source }\end{array}$} & \multirow[b]{2}{*}{ Substrate } & \multicolumn{3}{|c|}{$(R)$-Glucuronidation } & \multicolumn{3}{|c|}{ (S)-Glucuronidation } \\
\hline & & $K_{\mathrm{m}}(\mu \mathrm{M})$ & $\begin{array}{c}V_{\max }(\mathrm{pmol} / \\
\min / \mathrm{mg} \\
\text { protein })\end{array}$ & a & $K_{\mathrm{m}}(\mu \mathrm{M})$ & $\begin{array}{c}V_{\max }(\mathrm{pmol} / \\
\min / \mathrm{mg} \\
\text { protein) }\end{array}$ & a \\
\hline \multirow[t]{2}{*}{ HLM Lot A } & Racemate & $3.70 \pm 0.39$ & $13.7 \pm 2.4$ & $0.129 \pm 0.023$ & $4.18 \pm 0.85$ & $22.6 \pm 1.6$ & $0.795 \pm 0.022$ \\
\hline & Enantiomer & $17.1 \pm 7.3^{*}$ & $39.8 \pm 10.7 *$ & $0.712 \pm 0.038^{*}$ & $6.91 \pm 0.92 *$ & $26.5 \pm 3.8$ & $0.852 \pm 0.076$ \\
\hline \multirow[t]{2}{*}{ HLM Lot B } & Racemate & $6.57 \pm 0.31$ & $39.6 \pm 1.9$ & - & $13.2 \pm 0.84$ & $97.6 \pm 4.4$ & - \\
\hline & Enantiomer & $65.1 \pm 3.5^{* * *}$ & $274 \pm 5.1 * *$ & - & $32.2 \pm 2.6^{* * *}$ & $166 \pm 7.1^{* *}$ & - \\
\hline \multirow[t]{2}{*}{ HIM } & Racemate & $14.4 \pm 1.0$ & $14.8 \pm 1.2$ & - & $6.57 \pm 0.71$ & $2.65 \pm 0.72$ & - \\
\hline & Enantiomer & $26.1 \pm 3.2 * *$ & $24.4 \pm 1.1^{* * *}$ & - & $26.0 \pm 3.7^{*}$ & $6.58 \pm 0.26^{* * *}$ & - \\
\hline
\end{tabular}

The data of HLM Lot A were fitted to Michaelis-Menten and unsaturated component equation (Eq. 3). The data of HLM Lot B and HIM were fitted to MichaelisMenten equation (Eq. 1). Each value represents the estimate \pm S.D. of three independent measurements. ${ }^{*} p<0.05, * * p<0.01$ compared with racemate.

$$
V=\frac{V_{\max } \times[\mathrm{S}]}{\left(1+\frac{[\mathrm{S}]}{K_{\mathrm{m} \text { antipode }}}\right) \times\left(K_{\mathrm{m}}+[\mathrm{S}]\right)}
$$

\section{RESULTS}

Evaluation of Effects of Mutual Interaction between Carvedilol Enantiomers During Racemate Glucuronidation by HLM and HIM We investigated the concentration dependency of rates of glucuronidation of $(R)$ - and $(S)$-carvedilol by two lots of HLM and HIM and metabolic profiles by Eadie-Hofstee plot analysis. The results are shown in Fig. 2. Using racemic carvedilol as a substrate, we observed that the rate of $(S)$-carvedilol glucuronidation was higher than that of $(R)$-carvedilol glucuronidation by both HLM Lot A and Lot B. The rates of both $(R)-$ and $(S)$-carvedilol glucuronidation in the case of using each enantiomer as a substrate was higher than those in the case of using racemate as a substrate. However, the glucuronidation rate in Lot B was being saturated in high concentration range but that in Lot $\mathrm{A}$ was not. Moreover, Eadie-Hofstee plot analysis showed one compo- 


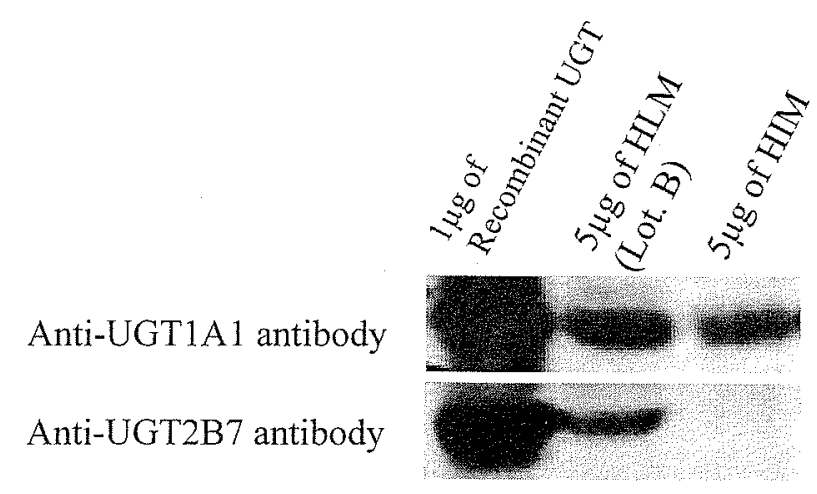

Fig. 4. Western Blot Analysis of HLM and HIM with Antisera against Human UGT1AI (Top) and UGT2B7 (Bottom)

Recombinant UGT1AI and UGT2B7 were used as positive controls.

nent in HLM Lot B and two distinct components in Lot A (Figs. 2C,D). On the other hand, the rate of $(R)$-carvedilol glucuronidation was superior to that of $(S)$-carvedilol by HIM in contrast to HLMs (Fig. 2). Moreover, this superiority of $(R)$-carvedilol glucuronidation was not changed by using enantiomer as a substrate, although the rates of $(R)$ - and $(S)$ carvedilol glucuronidation were higher than those in the racemate as substrate. Then kinetic parameters were calculated by curve fitting to Eqs. 1-3. Table 1 shows kinetic parameters of carvedilol glucuronidation by these enzyme sources. The data from HLM Lot A were fitted to the Michaelis-Menten and nonsaturated component equation (Eq. 3). The data from HLM Lot B and the HIM were fitted to Michaelis-Menten equation (Eq. 1). The all values of $V_{\max }$ in enantiomer except for $(S)$-glucuronidation by HLM Lot A were significantly higher than those in racemate (Table 1).

Figure 3 shows that the inhibitory effects of $(S)$-carvedilol on glucuronidation of $(R)$-carvedilol $(0.5 \mu \mathrm{M})$ by HLM Lot $\mathrm{A}$, Lot B and HIM as enzyme sources, and vice versa. (S)Carvedilol markedly reduced the rate of $(R)$-glucuronidation by both HLMs, and calculated $\mathrm{IC}_{50}$ values in Lot $\mathrm{A}$ and Lot $\mathrm{B}$ were $3.51 \pm 0.45 \mu \mathrm{M}$ and $10.4 \pm 0.5 \mu \mathrm{M}$, respectively. Inhibitory effects of $(R)$-carvedilol on $(S)$-glucuronidation were weaker than those of $(S)$-carvedilol, and calculated $\mathrm{IC}_{50}$ values in Lot $A$ and Lot B were $20.6 \pm 4.2 \mu \mathrm{M}$ and $260 \pm 107 \mu \mathrm{M}$, respectively. On the other hand, there was not so much difference in inhibitory effects between $(R)$ - and $(S)$-carvedilol in HIM $(8.82 \pm 2.1$, $16.5 \pm 1.1 \mu \mathrm{M})$.

We checked expressions of UGT1A1 and UGT2B7 in HLM and HIM by Western blotting (Fig. 4). The results showed that UGT2B7 was expressed in both HLMs and HIM but that UGT1A1 was expressed only in HLM.

Evaluation of Effects of Mutual Interaction between Carvedilol Enantiomers during Racemate Glucuronidation by UGT Isomers with Ability of Carvedilol Glucuronidation The rate of carvedilol glucuronide formation as a function of substrate concentration was measured using microsomes of $\mathrm{S} 9$ cells expressing recombinant UGT1A1, UGT2B4 and UGT2B7 (SUPERSOMES $\left.{ }^{(}\right)$, which were responsible for glucuronidation of carvedilol. ${ }^{7,2)}$ The results are shown in Figs. 5A,B for UGT1A1, in Figs. 5C,D for UGT2B4 and in Figs. 5E,F for UGT2B7. Kinetic parameters were calculated by using the Michaelis-Menten equation (Eq. 1) (Table 2).

$(R)$-Carvedilol was metabolized by UGT1A1 more rapidly than was $(S)$-carvedilol when the racemate or each enantiomer was used as a substrate (Figs. $5 \mathrm{~A}, \mathrm{~B}$ ). The ratios of $V_{\max }$ value of $(R)$-glucuronidation to $(S)$-glucuronidation $(R / S)$ were 7.3 (racemate) and 6.8 (enantiomer).

$(S)$-Carvedilol was metabolized by UGT2B 4 more rapidly than was $(R)$-carvedilol when the racemate was used as a substrate (Figs. $5 \mathrm{C}, \mathrm{D}$ ), and $R / S$ ratio was 0.56 . On the other hand, when each enantiomer was used as a substrate, $(R)$-carvedilol was metabolized much more rapidly than was $(S)$-carvedilol, and the $R / S$ ratio was 6.2 .

As for UGT2B7, (S)-carvedilol was metabolized more rapidly than was $(R)$-carvedilol when the racemate or each enantiomer was used as a substrate Figs. $5 \mathrm{E}, \mathrm{F}$, and $R / S$ ratios were 0.11 and 0.13 , respectively. Eadie-Hofstee plot analysis showed one component in all three UGT isoforms (data not shown).

Inhibitory effects of $(S)$-carvedilol on glucuronidation of $(R)$-carvedilol $(0.5 \mu \mathrm{M})$ by UGT1A1, UGT2B4 and UGT2B7 and vice versa are shown in Fig. 6. (S)-Carvedilol markedly reduced the rate of $(R)$-glucuronidation by UGT1A1, and the calculated $\mathrm{IC}_{50}$ value was $12.7 \pm 3.2 \mu \mathrm{M}$. Similarly, $(R)$-carvedilol reduced the rate of $(S)$-glucuronidation and the calculated $\mathrm{IC}_{50}$ value was $11.5 \pm 2.4 \mu \mathrm{M}$. (S)-Carvedilol reduced the rate of $(R)$-glucuronidation by UGT2B7, and the calculated $\mathrm{IC}_{50}$ was $59.0 \pm 8.6 \mu \mathrm{M}$. Similarly, $(R)$-carvedilol reduced the rate of $(S)$-glucuronidation, and the calculated $\mathrm{IC}_{50}$ was $57.1 \pm 4.5 \mu \mathrm{M}$. Inhibitory effects on glucuronidation by UGT2B7 were weak compared to those on glucuronidation by UGT1A1. On the other hand, as for UGT2B4, (S)-carvedilol markedly reduced the rate of $(R)$-glucuronidation, but the inhibitory effect of $(R)$-carvedilol on $(S)$-glucuronidation was very weak $\left(\mathrm{IC}_{50}\right.$ values: $6.6 \pm 1.1 \mu \mathrm{M}$ versus $\left.169 \pm 30 \mu \mathrm{M}\right)$.

Evaluation of the Contribution of Each UGT Isomer to Carvedilol Glucuronidation in HLM and HIM by the Inhibitory Effect of Mefenamic Acid Mefenamic acid is known as a typical inhibitor of UGT2B7. ${ }^{15)}$ We therefore examined the effects of mefenamic acid on carvedilol glucuronidation by not only UGT2B7 but also UGT1A1 and UGT2B4. Racemic carvedilol and $(R)$ - and $(S)$-carvedilol were used as substrates, and substrate concentrations were $0.8 \mu \mathrm{m}$ and $50 \mu \mathrm{M}$. The results are shown in Fig. $7(0.8 \mu \mathrm{M})$ and Fig. $8(50 \mu \mathrm{M})$.

There were differences in effects of mefenamic acid on carvedilol glucuronidation among the three UGT isoforms. Activity of carvedilol glucuronidation by UGT2B7 was decreased to about $20-50 \%$ of the control by $1 \mu \mathrm{M}$ of mefenamic acid, and it was decreased to $0-10 \%$ of the control by mefenamic acid at concentrations above $10 \mu \mathrm{M}$. UGT1A1 activity was inhibited mildly by mefenamic acid compared to the effect on UGT2B7 activity. In the presence of $10 \mu \mathrm{m}$ and $100 \mu \mathrm{m}$ mefenamic acid, activities of carvedilol glucuronidation were decreased to about $80 \%$ and $20 \%$ of the control, respectively. These tendencies of inhibition were observed in $(R)$-glucuronidation and (S)-glucuronidation by both UGT2B7 and UGT1Al with no dependence on substrate concentration. Moreover, UGT2B4 activity was hardly inhibited by low concentration of mefenamic acid compared to the effects on UGT1A1 activity. Namely, $100 \mu \mathrm{M}$ mefenamic acid had little inhibitory effect on activity of carvedilol glucuronidation. In the case of a substrate concentration of $0.8 \mu \mathrm{M}$, activities of carvedilol glucuronidation were decreased to $40-60 \%$ of the control in the presence of $500 \mu \mathrm{m}$ mefenamic acid. In the case 
UGT1A1
(A)
racemate

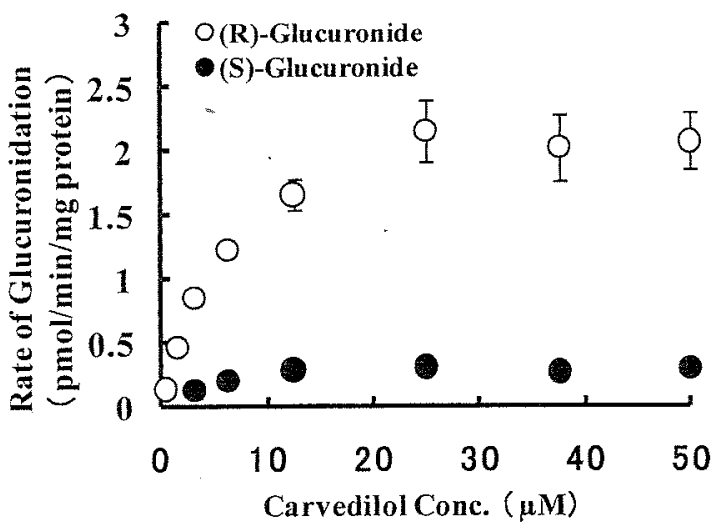

(B)

enantiomer

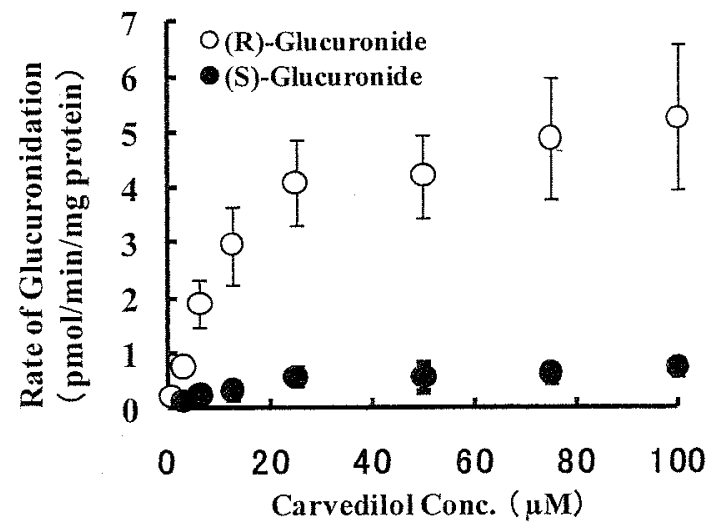

UGT2B4

(C)

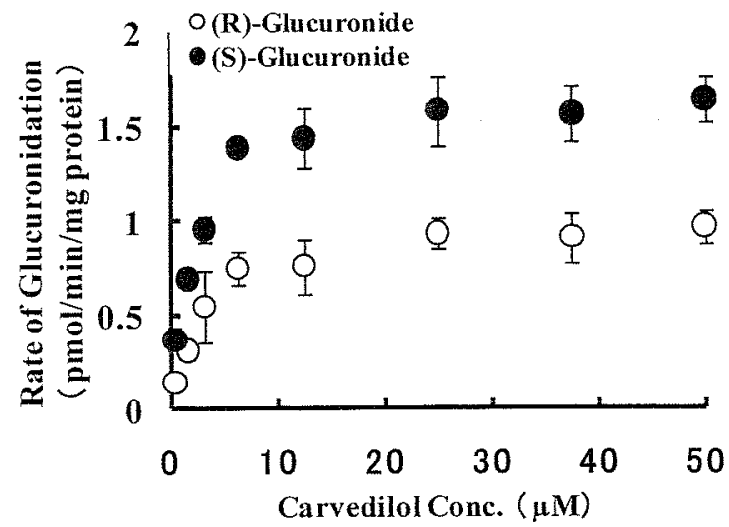

(D)

enantiomer

UGT2B7

(E)

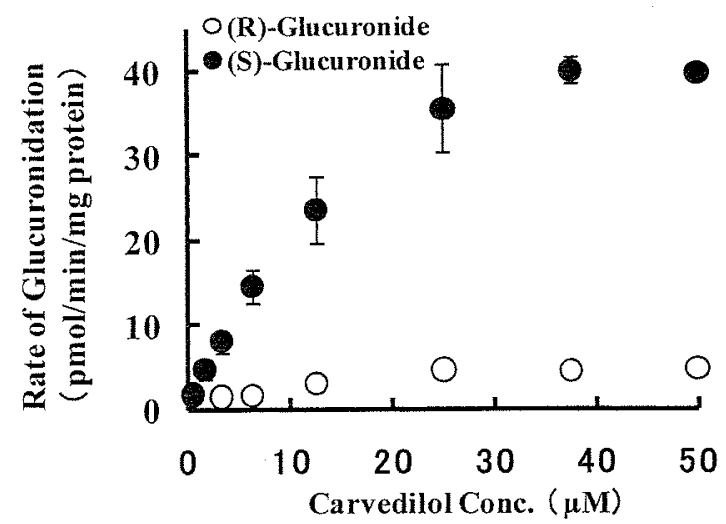

$$
\text { racemate }
$$

Carvedilol Conc. $(\mu \mathrm{M})$
(F)

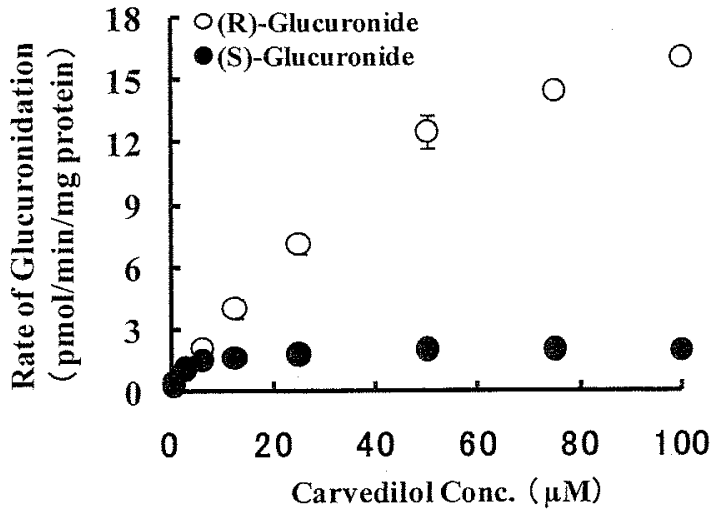

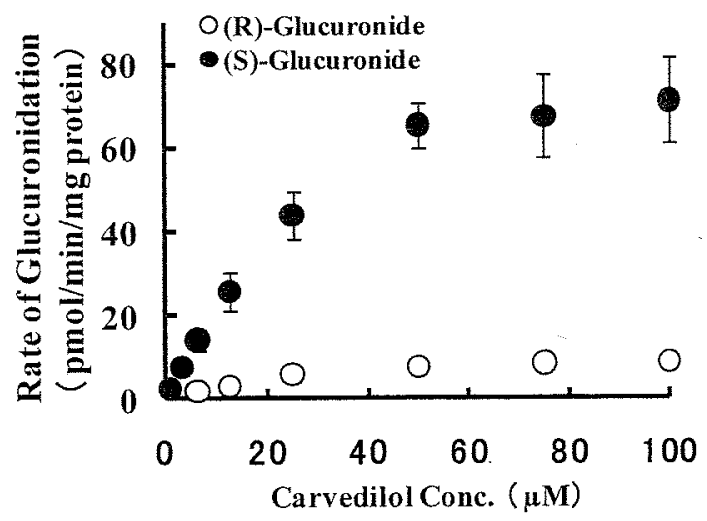

Fig. 5. Concentration Dependency of Glucuronidation Rate of (R)-and $(S)$-Carvedilol by Recombinant UGT1A1, UGT2B4 and UGT2B7

Racemate was used as a substrate in the left graph, and each enantiomer was used as a substrate in the right graph. Racemic carvedilol (0.4--50 $\mu \mathrm{M}$ as a single enantiomer concentration) and $(R)$ - or $(S)$-carvedilol $(0.8-100 \mu \mathrm{M})$ was incubated for $2 \mathrm{~h}$. Each point represents the mean $\pm S$.D. of three independent measurements.

of a substrate concentration of $50 \mu \mathrm{M}$, only $(R)$-glucuronidation from the $(R)$-enantiomer was decreased to about $40 \%$ of the control by $500 \mu \mathrm{M}$ mefenamic acid.
We then examined the effects of mefenamic acid on carvedilol glucuronidation by HLM Lot A, Lot B and HIM as well as recombinant UGTs. Substrate concentrations were 
Table 2. Kinetic Parameters of Carvedilol Glucuronidation by Recombinant UGT1A1, UGT2B4 and UGT2B7

\begin{tabular}{|c|c|c|c|c|c|}
\hline \multirow{2}{*}{ Enzyme source } & \multirow[b]{2}{*}{ Substrate } & \multicolumn{2}{|c|}{ (R)-Glucuronidation } & \multicolumn{2}{|c|}{ (S)-Glucuronidation } \\
\hline & & $K_{\mathrm{m}}(\mu \mathrm{M})$ & $\begin{array}{c}V_{\max } \\
\text { (pmol/min/mg protein) }\end{array}$ & $K_{\mathrm{m}}(\mu \mathrm{M})$ & $\begin{array}{c}V_{\max } \\
(\mathrm{pmol} / \mathrm{min} / \mathrm{mg} \text { protein) }\end{array}$ \\
\hline \multirow[t]{2}{*}{ UGT1A1 } & Racemate & $5.61 \pm 1.06$ & $2.39 \pm 0.32$ & $4.30 \pm 1.96$ & $0.328 \pm 0.018$ \\
\hline & Enantiomer & $1.40 \pm 1.1^{* *}$ & $5.92 \pm 1.31^{* * *}$ & $13.1 \pm 2.3^{* * *}$ & $0.900 \pm 0.170^{*}$ \\
\hline \multirow[t]{2}{*}{ UGT2B4 } & Racemate & $2.06 \pm 0.60$ & $0.934 \pm 0.122$ & $3.51 \pm 0.93$ & $1.67 \pm 0.18$ \\
\hline & Enantiomer & $79.4 \pm 8.4^{* *}$ & $29.6 \pm 1.4^{* *}$ & $3.09 \pm 0.12$ & $2.07 \pm 0.10$ \\
\hline \multirow[t]{2}{*}{ UGT2B7 } & Racemate & $9.31 \pm 1.78$ & $5.52 \pm 0.09$ & $12.3 \pm 3.4$ & $48.1 \pm 1.7$ \\
\hline & Enantiomer & $34.5 \pm 8.6^{*}$ & $13.4 \pm 0.8 * *$ & $33.0 \pm 2.4^{*}$ & $103 \pm 11 *$ \\
\hline
\end{tabular}

The data were fitted to the Michaelis-Menten model (Eq. 1). Each value represents mean \pm S.D. of three independent measurements. ${ }^{*} p<0.05, * * p<0.01$ compared with racemate.

(a) UGT1A1

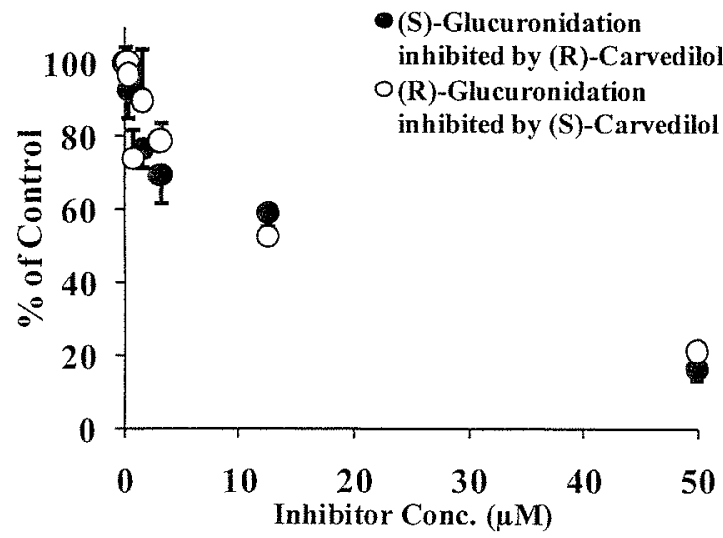

(b) UGT2B4

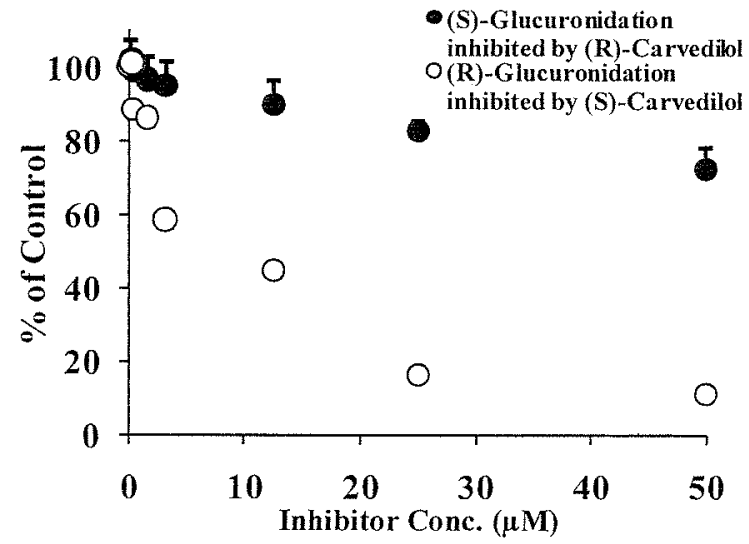

(c) UGT2B7

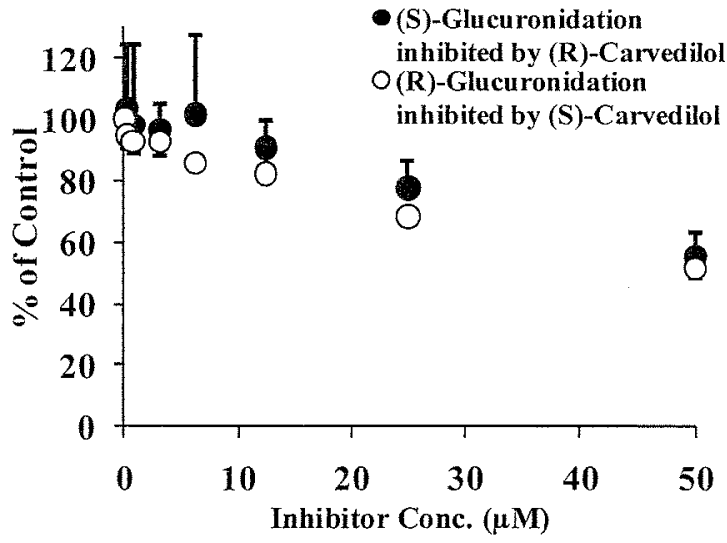

Fig. 6. Inhibitory Effects of Carvedilol Enantiomer on Glucuronidation of Its Antipode by UGT1Al (a), UGT2B4 (b), and UGT2B7 (c)

$(R)$ - or $(S)$-Carvedilol $(0.5 \mu \mathrm{M})$ as a sustrate was incubated in the absence and presence of its antipode (0.2-50 $\mu \mathrm{M})$ for $2 \mathrm{~h}$ (UGT1Al), 3h (UGT2B4) and $1 \mathrm{~h}$ (UGT2B7). Each point represents the mean $\pm S$.D. of three to four independent measurements.

$0.8 \mu \mathrm{M}$ and $50 \mu \mathrm{M}$, but the concentration was only $50 \mu \mathrm{M}$ in HIM because the amount of HIM with the same lot was not enough to examine at both $50 \mu \mathrm{M}$ and $0.8 \mu \mathrm{M}$. The results are shown in Fig. $7(0.8 \mu \mathrm{M})$ and Fig. $8(50 \mu \mathrm{M})$. When HLMs were used as enzyme sources, differences in inhibitory effects of mefenamic acid on carvedilol glucuronidation between Lot $A$ and Lot B were observed. Inhibitory effects of mefenamic acid on $(S)$-glucuronidation by Lot B were less than those by Lot $A$. In the presence of $500 \mu \mathrm{M}$ mefenamic acid, $20-30 \%$ of the activity of the control remained. This tendency was more prominent under a high substrate concentration $(50 \mu \mathrm{M})$ condi- tion.

As for HIM, activity of $(R)$-glucuronidation was decreased to about $90 \%$ of the control in the presence of $10 \mu \mathrm{M}$ mefenamic acid when both the racemate and each enantiomer were used as substrates, and $100 \mu \mathrm{m}$ mefenamic acid reduced the activity of $(R)$-glucuronidation to about $30 \%$ of the control. Activity of $(S)$-glucuronidation was decreased to $50-60 \%$ of the control in the presence of $10 \mu \mathrm{M}$ mefenamic acid, and $500 \mu \mathrm{M}$ mefenamic acid reduced the activity of $(S)$ glucuronidation to less than $10 \%$ of the control.

Application of Data Obtained in This Study to 
(A)

$(R)$-glucuronide (racemate $1.6 \mu \mathrm{M}$ )

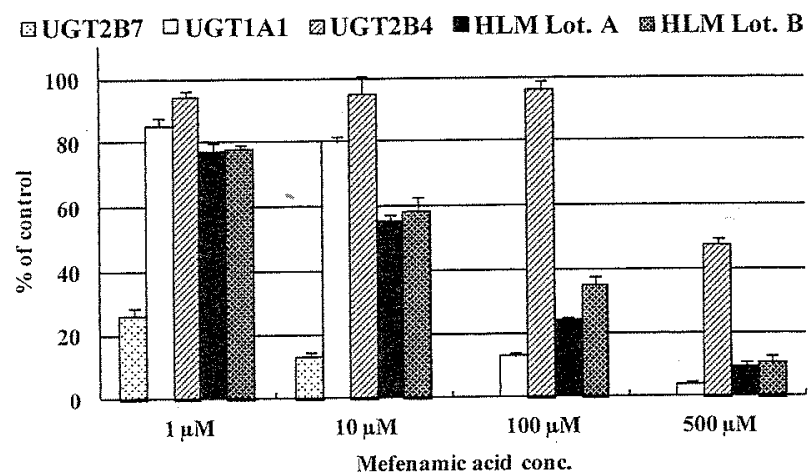

(C)

$(R)$-glucuronide (enantiomer $0.8 \mu \mathrm{M}$ )

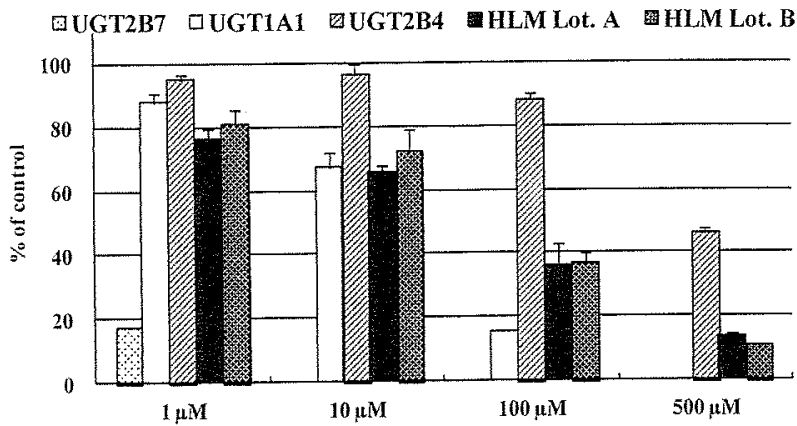

(B)

(S)-glucuronide (racemate $1.6 \mu \mathrm{M}$ )

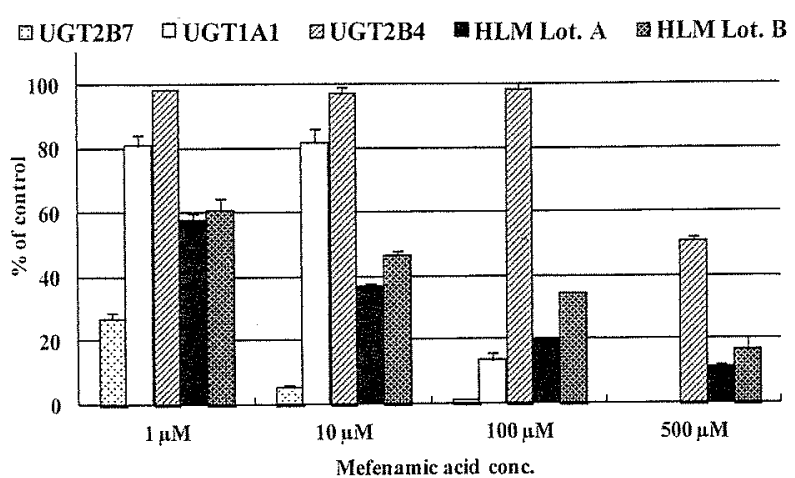

(D)

$(S)$-glucuronide (enantiomer $0.8 \mu \mathrm{M}$ )

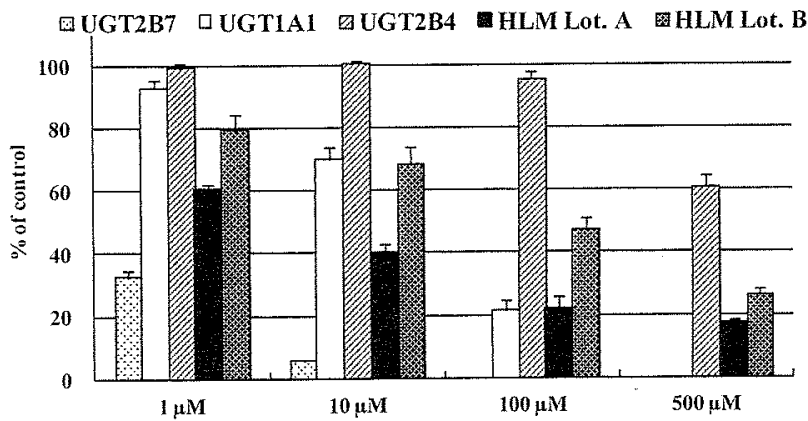

Fig. 7. Inhibitory Effects of Mefenamic Acid on the Formation of Carvedilol Glucuronide from a Low Concentration of Carvedilol $(0.8 \mu \mathrm{M}$ as a Single Enantiomer Concentration) by Recombinant UGT1A1, UGT2B4, UGT2B7, HLM Lot A, and Lot B

Each incubation time was $2 \mathrm{~h}$ (UGT1A1), $3 \mathrm{~h}$ (UGT2B4), $1 \mathrm{~h}$ (UGT2B7) and $30 \mathrm{~min}$ (both HLMs). Each column represents the mean with S.D. of three independent measurements.

Competitive and Noncompetitive Mutual Inhibition Michaelis-Menten Equations Then data obtained by using recombinant UGTs in this study (Table 2, enantiomer as a substrate) were fitted to Eqs. 6 and 7. As shown in Fig. 9, actual measured values of $(R)$-glucuronidation from racemate were fitted with competitive inhibition simulated curves in all UGT isoforms. On the other hand, actual measured values of $(S)$ glucuronidation from the racemate were fitted with competitive inhibition simulated curves in UGT $2 B 4$ and UGT2B7 as well as $(R)$-glucuronidation. However, in UGT1A1, these were not fitted with both competitive and non-competitive inhibition simulated curves.

\section{DISCUSSION}

We examined the concentration dependency and EadieHofstee plot analysis of glucuronidation rates of $(R)$ - and $(S)$-glucuronidation by HLMs and HIM in order to determine whether there were differences in enzyme kinetics and stereoselectivity among racemic carvedilol and each enantiomer as a substrate. In this study, we used two lots of HLM in order to confirm whether there are differences in carvedilol glucuronidation between pooled HLMs which were averaged by mixing HLMs from many individuals. Though HLM metabolized $(S)$-carvedilol to its glucuronide superior to $(R)$ carvedilol in the case of racemic carvedilol as a substrate in two lots, this superiority of $(S)$-carvedilol disappeared in the case of each enantiomer as a substrate, and the rates of both $(R)$ - and $(S)$-glucuronidation in the case of enantiomer as a substrate were higher than those in the case of the racemate (Figs. 2A,B). These results agreed with results of our previous study. ${ }^{12)}$ Moreover, values of $\mathrm{IC}_{50}$ indicated that $(R)$ - and $(S)$-enantiomers inhibit each other during racemate glucuronidation by HLM and that $(R)$-carvedilol glucuronidation was more easily inhibited by the antipode than was $(S)$-carvedilol glucuronidation (Figs. 3a,b). These results indicated that superiority of $(S)$-carvedilol glucuronidation by HLM was due to the difference in the inhibitory effect of each enantiomer on the other enantiomer during the racemate glucuronidation.

However, we observed differences in enzyme kinetics obtained from Eadie--Hofstee plot analysis between HLM Lot A and Lot B (Figs. 2C,D). Eadie-Hofstee plot of Lot A indicated the involvement of low-affinity and high-affinity enzymes (UGT isoforms) in carvedilol glucuronidation. It was thought that the enzyme of low affinity phase was not saturated in the range of substrate concentrations used in this study because the calculation diverged when using the two-enzyme Michaelis-Menten equation (Eq. 2), which is generally used for analyzing data from which Eadie-Hofstee plot analysis shows two distinct components. A feature of the enzyme kinetic parameters of Lot $A$ is a large difference in constant ' $a$,' which means a proportionality constant of non-saturated phase, between the racemate and $(R)$-enantiomer as a substrate (Table 1). Namely, one major enzyme in HLM Lot A was suspected to have a comparatively high value of $K_{\mathrm{m}}$, which is unsaturated at a substrate concentration of $100 \mu \mathrm{M}$, and to be easily inhibited by the $(S)$-enantiomer because value of "a" in the $(R)$-enantiomer was higher than that in the racemate and there 


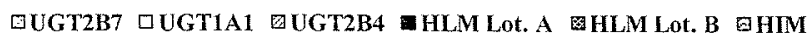

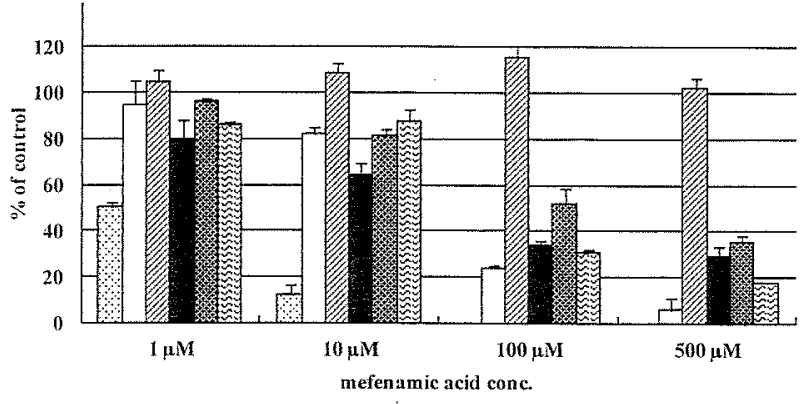

(C)

(R)-glucuronide (enantiomer $50 \mu \mathrm{M}$ )

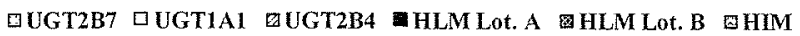

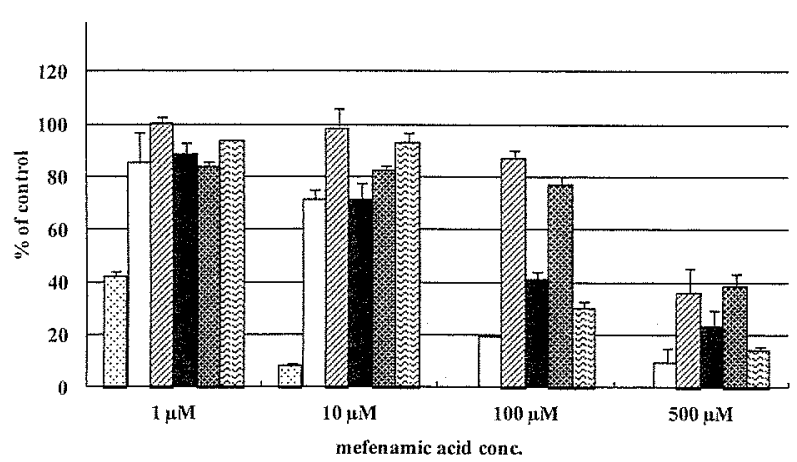

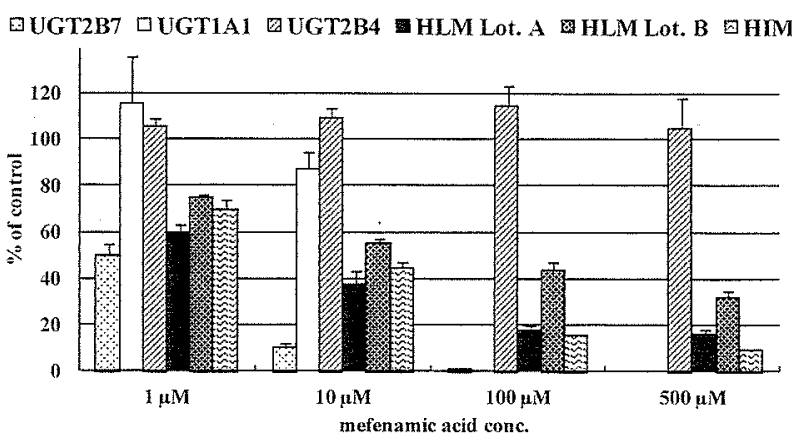

(D)

(S)-glucuronide (enantiomer $50 \mu \mathrm{M}$ )

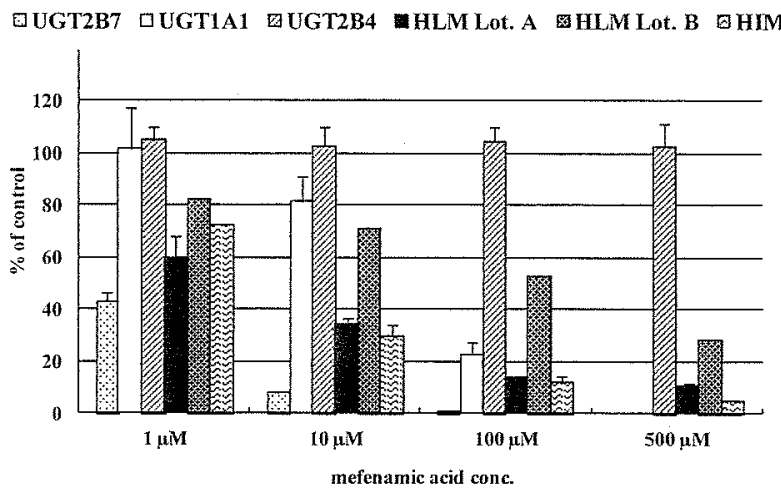

Fig. 8. Inhibitory Effects of Mefenamic Acid on the Formation of Carvedilol Glucuronide from a High Concentration of Carvedilol $(50 \mu \mathrm{M}$ as a Single Enantiomer Concentration) by Recombinant UGT1A1, UGT2B4, UGT2B7, HLM Lot A, Lot B and HIM

Each incubation time was $2 \mathrm{~h}$ (UGTIAl), $3 \mathrm{~h}$ (UGT2B4) and $1 \mathrm{~h}$ (UGT2B7 and HIM) and $30 \mathrm{~min}$ (both HLMs). Each column represents the mean with S.D. of three independent measurements.

wasn't difference in value of "a" between the $(S)$-enantiomer and the racemate as a substrate. Enzyme kinetic parameters of Lot B were obtained from curve fitting to Eq. 1, because of one component indicated by the Eadie-Hofstee plot (Figs. $2 \mathrm{C}, \mathrm{D}$ ). This result suggested that one or some enzymes with similar affinity for carvedilol were involved in carvedilol glucuronidation. A feature of the enzyme kinetic parameters of Lot B is a large difference in values of $K_{\mathrm{m}}$ and $V_{\max }$ between the racemate and $(R)$-enantiomer as a substrate (Table 1).

On the other hand, HIM preferred metabolizing $(R)$ - to $(S)$ carvedilol in the case of the racemate as a substrate, and this superiority of $(R)$-carvedilol glucuronidation was unchanged when each enantiomer was used as a substrate (Figs. 2A,B). However, the rates of both $(R)$ - and $(S)$-glucuronidation in the case of each enantiomer being used as a substrate were higher than those in the case of the racemate, and $\mathrm{IC}_{50}$ values indicated that there was no difference in inhibitory effect between $(R)$ - and $(S)$-carvedilol in HIM. Therefore, $(R)$ - and $(S)$ enantiomers might mutually inhibit glucuronidation by HIM similar to that by HLM, but it was thought that these inhibitions could not affect stereoselectivity of HIM for carvedilol. Ishida $e t a l$. reported that glucuronidation of $(S)$-carvedilol by HIM was also greater than that of $(R)$-carvedilol. ${ }^{13)}$ We then examined expressions of UGT1A1 and UGT2B7 proteins in HIM to determine the cause of this conflict between the results of this study and the results of previous Ishida's study. UGT2B4 highly expressed in liver but little or marginally expressed in small intestine by several reports. ${ }^{16-19)}$ On the other hand, it has reported that UGT1A1 and UGT2B7 expressed in both liver and small intestine, but interindividual variation in the expression level of UGT1A1 and UGT2B7 was found by using the RT-PCR method. ${ }^{16-20)}$ Therefore, we confirmed expression of UGT1A1 and UGT2B7 by Western blot analysis in this study. We have already reported that UGT1A1 and UGT2B7 have stereoselectivity for $(R)$ - and $(S)$-carvedilol, respectively. ${ }^{12)}$ UGT1A1 was detected in HIM used in this study, but UGT2B7 was not (Fig. 4). Therefore, it was thought that UGT2B7, which prefers metabolizing $(S)$-carvedilol, had little expression in HIM used in this study and that UGT1A1, which prefers metabolizing $(R)$-carvedilol, was expressed at a relatively high level in HIM. This difference might cause the superiority of $(R)$-carvedilol glucuronidation.

It has been reported that carvedilol is mainly metabolized by UGT1A1, UGT2B4 and UGT2B7 as phase II enzymes.7) Interindividuality of expression levels of UGT isoforms in various organs (liver, kidney, intestine, etc.) has been also reported. ${ }^{16.18)}$ In the case of using racemic carvedilol as a substrate, UGT1A1, UGT2B4 and UGT2B7 prefer metabolizing to $(R)$-carvedilol $(R: S=6: 1),(S)$-carvedilol $(R: S=1: 3)$ and $(S)$-carvedilol $(R: S=1: 9)$, respectively. ${ }^{712)}$ HLM is known to consist of various enzymes containing these three UGT isomers. Therefore, it is thought that the characteristics of stereoselectivity of HLM are determined by the composition of UGT isomers with different stereoselectivities of carvedilol. We then elucidated the properties of UGT1A1, UGT2B4 and UGT2B7 for carvedilol glucuronidation. In all three UGT iso- 


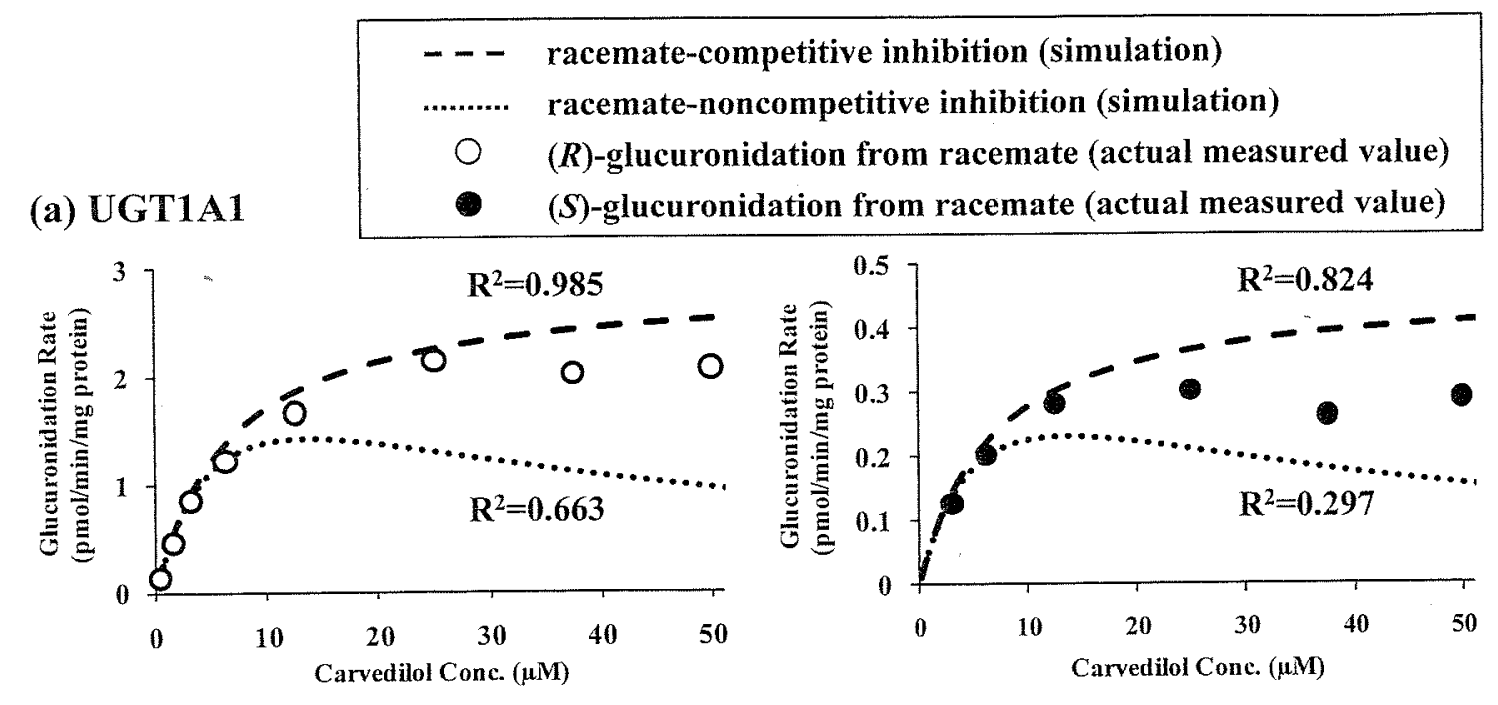

(b) UGT2B4
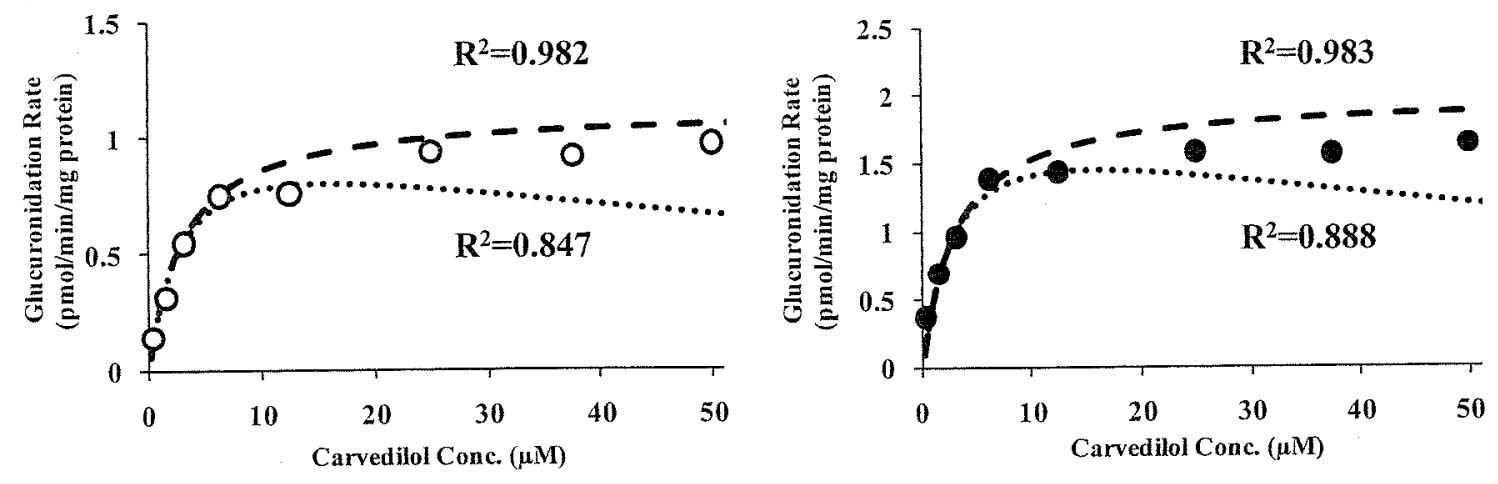

(c) UGT2B7
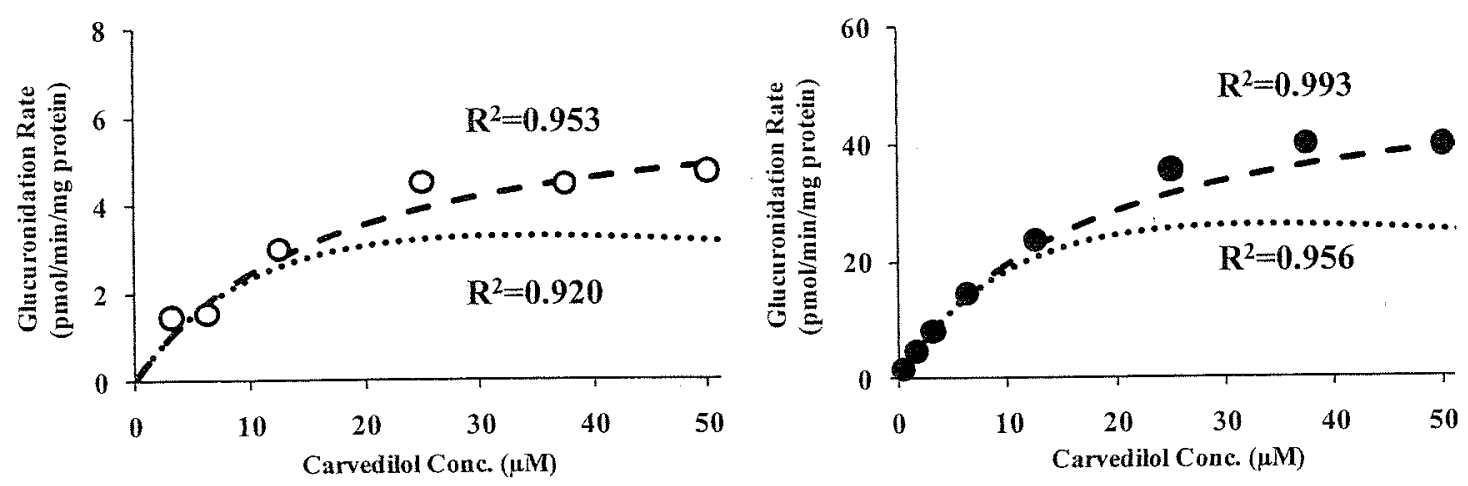

Fig. 9. Simulation Curves of Racemate-Competitive Inhibition Model (Eq. 4) and Racemate-Noncompetitive Inhibition Model (Eq. 5) in UGT1A1 (a), UGT2B4 (b) and UGT2B7 (c)

The graphs on the left and right represent $(R)$-glucuronidation and $(S)$-glucuronidation, respectively. Each value of $R^{2}$ represents coefficient of determination between actual measured values and simulated values.

mers, the rates of carvedilol glucuronidation in the case of the racemate used as a substrate were lower than those in the case of each enantiomer used as a substrate (Fig. 5). The results suggested mutual inhibitory effects between carvedilol enantiomers on glucuronidation mediated by all three UGT isomers. We observed changes in enzyme kinetic parameters and stereoselectivity in UGT2B4 depending on the kind of substrates. Namely, the rate of $(S)$-glucuronidation in the case of the racemate as a substrate by UGT2B 4 was higher than that of $(R)$-glucuronidation, but $(R)$-glucuronidation remarkably in- creased in the case of the $(R)$-enantiomer as a substrate and, in contrast, the rate of $(R)$-glucuronidation was much higher than that of $(S)$-glucuronidation (Figs. 5C,D). Values of $K_{\mathrm{m}}$ and $V_{\max }$ of $(R)$-glucuronidation in the case of the $(R)$-enantiomer as a substrate by UGT2B4 were greater than those in the case of the racemate (Table 2). Moreover, results for $\mathrm{IC}_{50}$ indicated that $(R)$-glucuronidation was much more easily inhibited by $(S)$-carvedilol than was $(S)$-glucuronidation by $(R)$-carvedilol in UGT2B4 ( $\mathrm{IC}_{50}: 6.6 \mu \mathrm{M}$ versus $\left.169 \mu \mathrm{M}\right)$. These changes in kinetic parameters and $\mathrm{IC}_{50}$ were similar to HLM Lot $\mathrm{B}$, and 
the content of UGT2B4 in each HLM was therefore presumed to contribute to differences in enzyme kinetics and stereoselectivity between HLM Lot A and Lot B. It was suspected that differences in values of constant ' $a$ ' between the racemate and $(R)$-enantiomer as a substrate in HLM Lot A reflected differences in values of $K_{\mathrm{m}}$ and $V_{\max }$ of $(R)$-glucuronidation in UGT2B4. On the other hand, stereoselectivities of UGT1A1 and UGT2B7 were not different between the racemate and each carvedilol as a substrate.

A specific inhibitor of each UGT isoform has not been identified clearly. We demonstrated that mefenamic acid could inhibit carvedilol glucuronidation by UGT1A1, UGT2B4 and UGT2B7 depending on its concentration. Namely, we clarified that mefenamic acid was able to completely inhibit catalytic activity of UGT2B 7 at $1-10 \mu \mathrm{M}$, almost completely inhibit catalytic activity of UGT1A1 at $100 \mu \mathrm{M}$, and partially inhibit catalytic activity of UGT2B4 at $500 \mu \mathrm{M}$ (Figs. 7, 8). These results suggested that mefenamic acid at various concentrations was able to inhibit carvedilol glucuronidation by UGT isoforms separately.

We then evaluated the contribution of each UGT isoform to carvedilol glucuronidation by HLM and HIM by inhibitory effects of mefenamic acid of various concentrations. Since the Eadie-Hofstee plot suggested two distinct components in HLM Lot A, we used substrate concentrations $(0.8,50 \mu \mathrm{M})$ (Figs. 7, 8). In HLM Lot A, no difference in results of inhibition profiles was observed in all experimental conditions of substrate type and substrate concentrations. Contribution ratios of UGT2B7, UGT1A1 and UGT2B4 for (R)-glucuronidation were estimated to be approximately $30-45 \%, 30 \%$ and $25-40 \%$, respectively. We expected that the contribution of UGTIAI would be greater because UGT1A1 was selective for $(R)$-carvedilol. However, the contribution of UGT1A1 was the same as or slightly less than that of UGT2B7, which was selective for $(S)$-carvedilol. This reason was probably that UGT2B7 could metabolize not only $(S)$-carvedilol but also $(R)$-carvedilol more greatly than could UGT1A1 despite UGT2B7 having stereoselectivity for $(S)$-carvedilol. Contribution ratios of UGT2B7, UGT1A1 and UGT2B4 for $(S)$-glucuronidation were estimated to be about $60-65 \%, 12-20 \%$ and $15-26 \%$, respectively. It is clarified that UGT2B7 was mainly responsible for $(S)$-carvedilol glucuronidation.

Inhibitory effects of mefenamic acid on carvedilol glucuronidation by Lot $B$ were less than those on carvedilol glucuronidation by Lot A. Apparently, the contribution of UGT2B4 was greater and the contribution of UGT2B7 was less in Lot B. Under the condition of $50 \mu \mathrm{M}$ of substrate, inhibition ratios of $(R)$-glucuronidation in the case of the racemate as a substrate were approximately $20 \%$ at an inhibitor concentration of $10 \mu \mathrm{M}$ and $50 \%$ at an inhibitor concentration of $100 \mu \mathrm{M}$. However, inhibition ratio of $(R)$-glucuronidation in the case of the $(R)$-enantiomer as a substrate was only $20 \%$ at an inhibitor concentration of $100 \mu \mathrm{m}$. These results indicated that $(R)$-carvedilol glucuronidation by UGT2B4 in HLM was inhibited by $(S)$-carvedilol in the case of using racemate as a substrate.

On the other hand, results for inhibitory effects of mefenamic acid on carvedilol glucuronidation by HIM suggested that UGT1A1 and UGT2B7 were mainly responsible for $(R)$-glucuronidation and $(S)$-glucuronidation by HIM, respec- tively. In this study, UGT2B7 was not detected by Western blot analysis. However, the contribution of UGT2B7 to $(S)$ glucuronidation by HIM might be relatively high despite the low expression level of UGT2B7 in HIM.

In this study, inhibition profiles of carvedilol glucuronidation by mefenamic acid with low $(0.8 \mu \mathrm{M})$ and high $(50 \mu \mathrm{m})$ substrate concentrations in HLM Lot A were not different, as above mentioned. This result conflicts with results of EadieHofstee plot analysis indicating two phase distinct components in HLM Lot A. The reason might be the effect of remaining enzyme activity in the presence of $500 \mu \mathrm{m}$ mefenamic acid. Five hundred micromolars of mefenamic acid could not completely inhibit carvedilol glucuronidation by recombinant UGT2B4. Therefore, the remaining enzyme activity in the presence of $500 \mu \mathrm{M}$ mefenamic acid in HLM was not necessarily activity of UGT2B4. Moreover, it has been reported that protein interaction between UGT and UGT or CYP in microsomes might cause changes in its enzyme activity and stereoselectivity. ${ }^{21-23)}$ The remaining enzyme activity might reflect this interaction. However, more research is needed to clarify the reason for this phenomenon.

UGT2B4 has been reported to be expressed in the liver at a level much higher than the level of UGT1A1 and UGT2B7. ${ }^{18,19)}$ However, there have been few reports of a major contribution of UGT2B4 to drug metabolism. In this study, we demonstrated that UGT2B4 played an important role in carvedilol glucuronidation by HLM. Values of $K_{\mathrm{m}}$, indicating affinity for an enzyme, were very different between $(R)$ - and $(S)$ glucuronidation in UGT2B4, though they were not different in UGT1A1 and UGT2B7. It has been reported that affinities of propranolol for UGT1A9 and UGT2B7 are not different between enantiomers, but those for UGT2B4 are different. ${ }^{24)}$ Therefore, UGT2B4 is thought to be greatly different in substrate recognition from other UGT isoforms.

Finally, we tried to construct an enzyme velocity equation that can account for the mutual inhibition observed in this study. Several models representing interaction between enantiomers have been reported. Kroemer et al. reported that a model equation based on methods of Segel ${ }^{25)}$ represents the sum of enzyme velocity for each enantiomer in the presence of both enantiomers at the same time. ${ }^{26)}$ Thotah et al. reported a multiple model equation combining competitive inhibition, two substrates, and a two-site model. ${ }^{27)}$ Enzyme kinetics under the condition of mutual interaction between enantiomers can be explained well by these equations. We constructed two model equations in order to understand more simply these interactions and to apply data obtained in this study to the two equations for each UGT isomer (Fig. 9). Actual measured data of $(R)$-glucuronidation from the racemate were better fitted to the competitive inhibition model than to the non-competitive inhibition model for all three UGT isoforms. All coefficients of determination in these UGT isoforms were 0.95 or more. On the other hand, actual measured data of $(S)$-glucuronidation from the racemate were also better fitted to the competitive inhibition model for UGT2B4 and UGT2B7, but the data were not fitted to either the competitive or non-competitive inhibition model for UGT1A1 (coefficient of determination: 0.824 versus 0.297 ). This might be due to self-inhibition at a high substrate concentration. These results suggested that the competitive mutual inhibition Michaelis-Menten equation (Eq. 5) is useful for understanding and evaluating interaction between 
Table 3. Kinetic Parameters for the Simulated Values of Racemate-Competitive Inhibition Model (Eq. 4) and Actual Measured Value

\begin{tabular}{|c|c|c|c|c|c|}
\hline \multirow[b]{2}{*}{ Enzyme source } & & \multicolumn{2}{|c|}{ (R)-Glucuronidation } & \multicolumn{2}{|c|}{$(S)$-Glucuronidation } \\
\hline & & $K_{\mathrm{m}}(\mu \mathrm{M})$ & $\begin{array}{c}V_{\max }(\mathrm{pmol} / \mathrm{min} / \mathrm{mg} \\
\text { protein })\end{array}$ & $K_{\mathrm{m}}(\mu \mathrm{M})$ & $\begin{array}{c}V_{\max }(\mathrm{pmol} / \mathrm{min} / \mathrm{mg} \\
\text { protein) }\end{array}$ \\
\hline \multirow{2}{*}{ UGTIA1 } & Measured value & $5.61 \pm 1.26$ & $2.39 \pm 0.25$ & $4.30 \pm 2.2$ & $0.328 \pm 0.02$ \\
\hline & -Simulated value & $6.77-$ & $2.86-$ & $6.77-$ & $0.46-$ \\
\hline \multirow{3}{*}{ UGT2B4 } & Measured value & $2.06 \pm 0.23$ & $0.934 \pm 0.096$ & $3.51 \pm 1.06$ & $1.67 \pm 0.04$ \\
\hline & Simulated value & $2.97-$ & $1.11-$ & $2.97-$ & $1.99-$ \\
\hline & Measured value & $9.31 \pm 2.13$ & $5.52 \pm 0.68$ & $12.3 \pm 2.5$ & $48.1 \pm 0.9$ \\
\hline UGT2B7 & Simulated value & $16.9-$ & $6.55-$ & $16.9-$ & $52.6-$ \\
\hline
\end{tabular}

The glucuronidation rate obtained from simulation of Eq. 4 was fitted to Michaelis-Menten equation (Eq. 1). The data of actual measured value were reprinted from Table 3. Each value represents mean \pm S.D. of three independent measurements.

enantiomers in UGT2B4 and UGT2B7.

In conclusion, we demonstrated that racemic carvedilol glucuronidation by HLM was affected by mutual inhibition between the enantiomers and that superiority of $(S)$-carvedilol glucuronidation by HLM was due to the difference in the inhibitory effect of each enantiomer on the other enantiomer during the racemate glucuronidation. Moreover, we suggested that the contribution of each UGT isoform to carvedilol glucuronidation by HLM and HIM was able to be evaluated by inhibitory effects of mefenamic acid of various concentrations. Especially, UGT2B4, in which $(R)$-glucuronidation was much more easily inhibited by $(S)$-carvedilol than was $(S)$ glucuronidation by $(R)$-carvedilol, was important role to characterize the property of carvedilol glucuronidation by HLM during racemate glucuronidation.

Acknowlegements This reseach was supported financially by Kuribayashi Scientific Scholarship Foundation, Hokkaido, Japan. We thank Aki Ootomo for expert secretarial assistance.

\section{REFERENCES}

1) Brocks DR. Drug disposition in three dimensions: an update on stereoselectivity in pharmacokinetics. Biopharm. Drug Dispos., 27, 387-406 (2006).

2) Nakano $M$, Higaki K, Kawahara S. Enantiomer-enantiomer interaction of a uricosuric antihypertensive diuretic (DBCA) in renal tubular secretion and stereoselective inhibition by probenecid in the cynomolgus monkey. Xenobiotica, 23, 525-536 (1993).

3) Desta Z, Soukhova N, Morocho AM, Flockhart DA. Stereoselective metabolism of cisapride and enantiomer-enantiomer interaction in human cytochrome P450 enzymes: major role of CYP3A. J. Pharmacol. Exp. Ther., 298, 508-520 (2001).

4) von Möllendorff E, Abshagen U, Akpan W, Neugebauer G, Schröter E. Clinical pharmacologic investigations with carvedilol, a new $\beta$-blocker with direct vasodilator activity. Clin. Pharmacol. Ther, 39, 677-682 (1986).

5) Frishman WH. Carvedilol. N. Engl. J. Med., 339, 1759-1765 (1998).

6) Oldham HG, Clarke SE. In vitro identification of the human cytochrome P450 enzymes involved in the metabolism of $R(+)$ - and S(-)-carvedilol. Drug Metab. Dispos., 25, 970-977 (1997).

7) Ohno A, Saito $Y$, Hanioka N, Jinno H, Saeki M, Ando M, Ozawa $S$, Sawada J. Involvement of human hepatic UGT1A1, UGT2B4, and UGT2B7 in the glucuronidation of carvedilol. Drug Metab. Dispos., $32,235-239$ (2004).

8) Zhou HH, Wood AJ. Stereoselective disposition of carvedilol is determined by CYP2D6. Clin. Pharmacol. Ther., 57, 518-524 (1995).

9) Giessmann $T$, Modess C, Hecker U, Zschiesche M, Dazert P,
Kunert-Keil C, Warzok R, Engel G, Weitschies W, Cascorbi I, Kroemer HK, Siegmund W. CYP2D6 genotype and induction of intestinal drug transporters by rifampin predict presystemic clearance of carvedilol in healthy subjects. Clin. Pharmacol. Ther., 75, $213-222(2004)$.

10) Honda $M$, Nozawa $T$, Igarashi $N$, Inoue $H$, Arakawa $R$, Ogura $Y$, Okabe $H$, Taguchi $M$, Hashimoto Y. Effect of CYP2D6*10 on the pharmacokinetics of $R$ - and $S$-carvedilol in healthy Japanese volunteers. Biol. Pharm. Bull., 28, 1476-1479 (2005).

11) Honda $M$, Ogura $Y$, Toyoda $W$, Taguchi $M$, Nozawa $T$, Inoue $H$, Hashimoto $Y$. Multiple regression analysis of pharmacogenetic variability of carvedilol disposition in 54 healthy Japanese volunteers. Biol. Pharm. Bull., 29, $772-778$ (2006).

12) Takekuma $Y$, Takenaka $T$, Yamazaki $K$, Ueno $K$, Sugawara $M$. Stereoselective metabolism of racemic carvedilol by UGT1Al and UGT2B7, and effects of mutation of these enzymes on glucuronidation activity. Biol. Pharm. Bull., 30, 2146-2153 (2007).

13) Ishida $\mathrm{K}$, Taira $\mathrm{S}$, Morishita $H$, Kayano $Y$, Taguchi $M$, Hashimoto $Y$. Stereoselective oxidation and glucuronidation of carvedilol in human liver and intestinal microsomes. Biol. Pharm. Bull., 31, 1297-1300 (2008).

14) Takekuma Y, Takenaka T, Kiyokawa M, Yamazaki K, Okamoto $H$, Kitabatake A, Tsutsui $H$, Sugawara $M$. Evaluation of effects of polymorphism for metabolic enzymes on pharmacokinetics of carvedilol by population pharmacokinetic analysis. Biol. Pharm. Bull., 30, 537-542 (2007).

15) Mano $Y$, Usui $T$, Kamimura $H$. Inhibitory potential of nonsteroidal anti-inflammatory drugs on UDP-glucuronosyltransferase $2 \mathrm{~B} 7$ in human liver microsomes. Eur. J. Clin. Pharmacol., 63, 211-216 (2007).

16) Strassburg CP, Kneip S, Topp J, Obermayer-Straub P, Barut A, Tukey RH, Manns MP. Polymorphic gene regulation and interindividual variation of UDP-glucuronosyltransferase activity in human small intestine. J. Biol. Chem., 275, 36164-36171 (2000).

17) Nakamura A, Nakajima $M$, Yamanaka $H$, Fujiwara $R$, Yokoi $T$. Expression of UGT1A and UGT2B mRNA in human normal tissues and various cell lines. Drug Metab. Dispos., 36, 1461-1464 (2008).

18) Ohno S, Nakajin S. Determination of mRNA expression of human UDP-glucuronosyltransferases and application for localization in various human tissues by real-time reverse transcriptase-polymerase chain reaction. Drutg Metab. Dispos., 37, 32-40 (2009).

19) Izukawa $T$, Nakajima $M$, Fujiwara R, Yamanaka H, Fukami $T$, Takamiya M, Aoki Y, Ikushiro S, Sakaki T, Yokoi T. Quantitative analysis of UDP-glucuronosyltransferase (UGT) $1 \mathrm{~A}$ and UGT2B expression levels in human livers. Drug Metab. Dispos., 37, 17591768 (2009).

20) Nishimura $M$, Naito $S$. Tissue-specific mRNA expression profiles of human phase I metabolizing enzymes except for cytochrome P450 and phase II metabolizing enzymes. Drug Metab. Pharmacokinet., 21, 357-374 (2006).

21) Ishii $Y$, Iwanaga $M$, Nishimura $Y$, Takeda $S$, Ikushiro $S$, Nagata 
K, Yamazoe Y, Mackenzie PI, Yamada H. Protein-protein interactions between rat hepatic cytochromes P450 (P450s) and UDPglucuronosyltransferases (UGTs): evidence for the functionally active UGT in P450-UGT complex. Drug Metab. Pharmacokinet., 22, 367-376 (2007).

22) Nakajima $M$, Yamanaka $H$, Fujiwara $R$, Katoh $M$, Yokoi $T$. Stereoselective glucuronidation of 5-(4'-hydroxyphenyl)-5-phenylhydantoin by human UDP-glucuronosyltransferase (UGT) 1A1, UGT1A9, and UGT2B15: effects of UGT-UGT interactions. Drug Metab. Dispos., 35, 1679-1686 (2007).

23) Ishii $Y$, Takeda S, Yamada H. Modulation of UDP-glucuronosyltransferase activity by protein-protein association. Drug Metab. Rev, 42, 145-158 (2010).

24) Hanioka N, Hayashi K, Shimizudani T, Nagaoka K, Koeda A, Naito
S, Narimatsu S. Stereoselective glucuronidation of propranolol in human and cynomolgus monkey liver microsomes: role of human hepatic UDP-glucuronosyltransferase isoforms, UGT1A9, UGT2B4 and UGT2B7. Pharmacology, 82, 293-303 (2008).

25) Segel IH. Enzyme Kinetics. John-Wiley \& Sons, New York, pp. $113-118$ (1975).

26) Kroemer HK, Fischer C, Meese CO, Eichelbaum M. Enantiomer/ enantiomer interaction of $(S)$ - and $(R)$-propafenone for cytochrome P4501ID6-catalyzed 5-hydroxylation: in vitro evaluation of the mechanism. Mol. Pharmacol., 40, 135-142 (1991).

27) Totah RA, Allen KE, Sheffels P, Whittington D, Kharasch ED. Enantiomeric metabolic interactions and stereoselective human methadone metabolism. J. Pharmacol. Exp. Ther, 321, 389-399 (2007). 